\title{
Measuring healthcare quality
}

\section{Condition or procedure-specific versus aggregate performance}

Historically, healthcare performance assessment focused on outcomes for specific procedures and conditions. However, sample sizes are often inadequate, and it is difficult to assess overall hospital performance from a few diagnoses. Consequently, some have advocated measures such as the Hospital Standardized Mortality Ratio (HSMR) that aggregate results from many diagnoses at an institution. Recent studies show that the results of such metrics vary substantially depending on eligibility and exclusion criteria. The latter should focus on those conditions where there is the strongest association between quality of care delivered and outcomes.

\section{Timing of outcomes measurement} $\nabla$

Many quality measurement programs determine outcomes at the time of hospital discharge. However, in regions where there are few extended care facilities, patients remain in their initial hospital longer, and complications and deaths are more likely to be captured during the index hospitalization. To mitigate this potential bias, many advocate use of fixed time periods, such as 30-days following admission or procedure, regardless of venue.

For some diseases such as cancer, the impact of quality of care may not be apparent in the shortterm and may require longer-term follow-up.

\section{Types of measures}

Structural measures assess the infrastructure features that facilitate high quality care, such as electronic health records. Process measures assess whether patients receive care consistent with evidence-based guidelines. Such measures are easily captured and do not require risk adjustment, but they must have carefully specified inclusion and exclusion criteria. Some studies have questioned whether process measure compliance results in better outcomes. Outcome measures integrate the net effect of measured and unmeasured processes of care, and they are of greatest interest to patients. However, they require risk-adjustment (which is best done with clinical data) and adequate sample sizes.

\section{Composite measures}

$\nabla$

Measurement of single metrics provides an incomplete assessment of quality. The Institute of Medicine recommends expanded use of multidimensional composite measures that provide a more comprehensive quality perspective. An example is the Society of Thoracic Surgeons CABG Composite score, which includes 11 process and outcomes measures within 4 domains (risk adjusted mortality and morbidity; use of the internal mammary artery; and use of recommended medications).

\section{Public reporting}

It is hypothesized that public reporting of healthcare outcomes improves quality and increases the market share of better providers. Although public reporting has been associated with improved survival, some results suggest that other methods such as confidential provider feedback and dissemination of best-practices are equally effective.

Report cards have not substantially impacted choice of providers or market share in the past, but this may relate to consumer lack of awareness or their inability to understand report cards. As these problems are addressed through consumer education and more interpretable report card designs, the impact on consumer choice may increase.

The main justification for public reporting is to promote patient autonomy. This is the right of patients to make informed decisions about their choice of treatment or provider based on all available data, including performance results.

The major unintended negative consequence of public report cards is risk aversion. When their results are publicly reported, providers may refuse to accept high-risk patients. Despite risk-adjustment, providers may not believe that the severity of their patients will be taken into account, thereby adversely affecting their reported results. Numerous methods have been suggested to mitigate risk aversion, including better risk adjustment, separate reporting of high-risk patients, or exclusion of high-risk patients from reporting.

Author's declaration: No conflict of interest.
D. M. Shahian

Qualitätsmanagement

Keywords

quality measurement

Outcome

public reporting

Schlüsselwörter

Qualitätsmessung

Ergebnisqualität

Qualitätstransparenz

Institut

Massachusetts General Hospital, Harvard Medical School, Center for Quality and Safety and Department of Surgery, Boston, USA

Bibliografie DOI $10.1055 / \mathrm{s}-0031-1286086$ Dtsch Med Wochenschr 2011 136: S57 . (c) Georg Thieme Verlag KG Stuttgart - New York . ISSN 0012-0472

\section{Korrespondenz}

\section{David M. Shahian, MD}

Massachusetts General Hospita Harvard Medical School Center for Quality and Safety and Department of Surgery 55 Fruit St

Boston, MA 02114, USA Tel. +1 6176434335 eMail dshahian@partners.org 